Tôhoku Math. Journ.

31 (1979), 165-178.

\title{
ON THE GAUSS-BONNET FORMULA ON ODD-DIMENSIONAL MANIFOLDS
}

\author{
A. Reventós
}

(Received February 2, 1978, revised April 17, 1978)

1. Introduction. This work can be considered as a continuation of "A formula on some odd-dimensional Riemannian manifolds related to the Gauss-Bonnet formula" by S. Tanno [7].

Let $B$ be a compact orientable Riemannian manifold of dimension $2 n$. The Gauss-Bonnet-Chern formula says

$$
\int_{B} Q=2^{2 n} \pi^{n} n ! \chi(B)
$$

where $Q$ denotes the Gauss-Bonnet form and $\chi(B)$ is the Euler-Poincaré characteristic. In this formula even dimensionality is essential. If $B$ is odd-dimensional, then $\chi(B)=0$. However, for odd-dimensional manifolds, we can think on the possibility of finding an analogous formula in which the right hand side is a certain expression containing the Betti numbers.

On trying to find a formula analogous to (1) we expect the nonvanishing fields on the manifold to play an important role.

On this line, S. Tanno [7] found the following result:

THEOREM. Let $\left(E^{2 n+1}, g\right)$ be a compact Riemannian manifold having a unit Killing vector field $\xi$ such that $R(X, \xi) Y=g(X, Y) \xi-g(Y, \xi) X$, for any $X, Y$ vector fields on $E^{2 n+1}$ (i.e., a Sasakian manifold). Then, if the field $\xi$ is regular (Palais [5]), we have

$$
\left((-1)^{n} / \ell(\xi) 2^{2 n} \pi^{n} n !\right) \int_{E} F(\Omega, \xi)=\sum_{r=0}^{n}(n+1-r)(-1)^{r} b_{r}(E),
$$

where $b_{i}(E)$ is the $i$-th Betti number of $E$ and $F(\Omega, \xi)$ is an expression which depends on the curvature $\Omega$ and the field $\xi$.

We want to obtain an expression like (2) for odd-dimensional Riemannian manifolds, which are not necessarily Sasakian.

However it seems convenient to continue assuming $\xi$ to be a regular unit Killing vector field. Concretely we state the following problem.

Let $E$ be a compact manifold of dimension $2 n+1$ with a regular field $\xi$ on $E$. Let $g$ be a Riemannian metric on $E$ for which $\xi$ is a unit Killing vector field. We want to find under these assumptions a formula 
analogous to (2). The simplest example of a manifold satisfying our hypothesis and not Tanno's is the following:

Let $B$ be a compact orientable Riemannian manifold of dimension $2 n$. Let us suppose that $b_{1}(B)$ is even and $b_{0}(B)=1$. We consider the product $B \times S^{1}$. A unit tangent field to $S^{1}$ defines a unit regular Killing vector field on $B \times S^{1}$, related to the product metric. So, $B \times S^{1}$ satisfies our hypothesis, but since $b_{1}\left(B \times S^{1}\right)$ is odd, on $B \times S^{1}$ there is not any Sasakian structure because it is well known that the first Betti number of a Sasakian manifold is zero or even.

The first result which we obtain is the following.

THEOREM 1. Let $(E, \xi, g)$ be a compact orientable Riemannian manifold of dimension $2 n+1$, with $\xi$ a regular unit Killing vector field on $E$. Then

$$
\begin{aligned}
& \left((-1)^{n} / \ell(\xi) 2^{2 n} \pi^{n} n !\right) \int_{E} f(\Omega, \xi)=\sum_{r=0}^{n}(n+1-r)(-1)^{r} b_{r}(E) \\
& \quad+\sum_{r=0}^{n-1}(-1)^{r} d_{r},
\end{aligned}
$$

where $f(\Omega, \xi)$ is a certain function of $\Omega$ and $\xi . \quad b_{r}(E)$ is the $r$-th Betti number of $E$ and $d_{r}=\operatorname{dim} \operatorname{Ker}\left\{H^{r}(E / \xi, R) \rightarrow H^{r+2}(E / \xi, R)\right\}$. This map is the multiplication by the Euler class of the bundle $E \rightarrow E / \xi$.

The left hand side of (3) depends on $g$ and $\xi$. However the right hand side depends only on $\xi$ (because $d_{r}$ depends on $\xi$ ). The right hand side is concretely $\chi(E / \xi)$.

Nevertheless, it is possible that, in the most interesting cases, the conditions over the field $\xi$ (unit Killing regular) determine $\chi(E / \xi)$. That is, if $\xi$ and $\xi^{\prime}$ are two unit Killing regular vector fields on $E$, then $\chi(E / \xi)=\chi\left(E / \xi^{\prime}\right)$. In this case the right hand side of (3) could be calculated knowing only the Betti numbers of $E$.

In this work, we have tried to solve these problems in low dimensions (dim 3 and $\operatorname{dim} 5)$ and we have obtained some particular results for higher dimensions.

I am indebted to Professor J. Girbau who suggested this problem to me and helped in some particular cases.

I also wish to express my sincere gratitude to Professor S. Tanno for an invaluable review of my manuscript and his commentaries on Corollary 2.

2. Preliminaries and notations. To state our result in dimension 5 more easily we introduce the following definition. 
Definition. Let $E$ be a compact connected manifold of dimension 5 . We say that the Betti numbers $b_{1}(E)$ and $b_{2}(E)$ satisfy the $R_{1}$-condition if they satisfy any of the following conditions:

(1) $b_{1}(E)=0, b_{2}(E)$ arbitrary

(2) $b_{1}(E)=1, b_{2}(E)=0$

(3) $b_{1}(E)$ even $\neq 2, b_{2}(E)=1$

(4) $b_{1}(E)=2, b_{2}(E)=0$ or 1 .

And we say that they satisfy the $R_{2}$-condition if

( 5 ) $b_{1}(E)$ odd $\neq 1$, and $b_{2}(E)<12\left[\left(4 b_{1}(E)-4\right) / 10\right]+12 \delta_{01}+3-4 b_{1}(E)$. $\left(\delta_{01}=0\right.$ if $\left(\left(4 b_{1}(E)-4\right) / 10\right) \in Z$, and $\delta_{01}=1$ otherwise. The bracket is the Gauss symbol meaning the "integer part").

We give now a table which shows, for low Betti numbers, which ones satisfy the $R_{2}$-condition.

\begin{tabular}{|c|c|}
\hline$b_{1}(E)$ & $b_{2}(E)$ \\
\hline 3 & $0,1,2$. \\
\hline 5 & $0,1,2,3,4,5,6$ \\
\hline 7 & $0,1,2,3,4,5,6,7,8,9,10$ \\
\hline$\cdot$ & $\cdot$ \\
\hline
\end{tabular}

In the proof of Theorem 3, we use essentially the classification of complex surfaces given by Kodaira [3]. Let us recall the notation, definitions and some results.

Let $S$ be a compact complex manifold of complex dimension 2 . We denote by $c_{1}$ and $c_{2}$ the first and second Chern classes of $S$. We represent any cohomology class $c \in H^{4}(S, Z)$ by the value $c(S)$ of $c$ on the fundamental cycle of the surface $S$ oriented in the natural way with respect to its complex structure. Thus we consider $c_{1}^{2}$ and $c_{2}$ as integers.

We define $h^{r, s}=\operatorname{dim} H^{s}\left(S, \Omega^{r}\right)$ where $\Omega^{r}$ is the sheaf over $S$ of germs of holomorfic $r$-forms. We set $p_{g}=h^{2,0}=h^{0,2}$ and $q=h^{0,1}$ and we call $p_{g}$ and $q$, respectively, the geometric genus and the irregularity of $S$.

Moreover, if $\xi$ and $\eta$ are closed 2-forms on $S$ we define

$$
(\xi, \eta)=\int_{S} \xi \wedge \eta
$$

We may consider (, ) as a non-singular symmetric bilinear form defined on $H^{2}(S, \boldsymbol{R})$. We define $b^{+}$and $b^{-}$to be respectively the numbers of positive and negative eigen values of the symmetric bilinear form.

Kodaira obtains the following theorem, which we call

THEOREM A. If $b_{1}$ is even, then $2 q=b_{1}, 2 p_{g}=b^{+}-1, h^{1,0}=q . \quad$ If 
$b_{1}$ is odd, then $2 q=b_{1}+1,2 p_{g}=b^{+}, h^{1,0}=q-1$. Moreover $c_{1}^{2}+8 q+$ $b^{-}=10 p_{g}+8$ if $b_{1}$ is odd, and $c_{1}^{2}+8 q+b^{-}=10 p_{g}+9$ if $b_{1}$ is even.

We use also the following theorem (Grauert):

"Any surface is obtained from a surface containing no exceptional curve by means of a finite number of quadratic transformations".

Let us recall that an exceptional curve (of the first kind) on $S$ is, by definition, a non-singular rational curve $C$ with $\left(C^{2}\right)=-1$.

Kodaira gives a proof of this theorem by showing that an exceptional curve (of the first kind) $C$ on $S$ is contractible, i.e., there exists $f: S \rightarrow S^{\prime}$ with $f(C)=p, S-C$ isomorphe to $S^{\prime}-p$, and $f$ is a monoidal transformation.

We have $H_{2}(S, \boldsymbol{Z})=H_{2}\left(S^{\prime}, \boldsymbol{Z}\right) \oplus \boldsymbol{Z}$. Since $H_{2}(S, \boldsymbol{Z})$ is a finitely generated $\boldsymbol{Z}$-module, in the classification of surfaces, we always assume that the surfaces do not contain exceptional curves.

From the last relation we have $c_{1}^{2}(S)=c_{1}^{2}\left(S^{\prime}\right)-1$ which for a finite number $n_{t}$ of quadratic transformations is $c_{1}^{2}(S)=c_{1}^{2}\left(S^{\prime}\right)-n_{t}$.

We also use the following theorem of Kodaira, which we call

THEOREM B. We have the following table for surfaces which are free from exceptional curves:

\begin{tabular}{cccccc}
$b_{1}$ & $P_{12}$ & $P_{2}$ & $K$ & $c_{1}^{2}$ & structure \\
\hline even & 0 & 0 & & & plane or ruled \\
0 & 1 & 1 & $=0$ & 0 & K3 surface \\
4 & 1 & 1 & $=0$ & 0 & complex torus \\
even & + & & $\neq 0$ & 0 & elliptic \\
even & + & + & & + & algebraic \\
odd & + & & & 0 & elliptic \\
1 & 0 & 0 & & & $?$
\end{tabular}

where $P_{m}=\operatorname{dim} H^{\circ}\left(S, \Omega^{\circ}(m K)\right)$ and $K$ is a canonical divisor.

Finally we recall the following result (Kodaira).

THEOREM C. If on a complex surface we have $p_{g}=0$ and $c_{1}^{2}>0$, then $q=0$.

3. Proof of Theorem 1. Let $E$ be a compact orientable manifold of dimension $2 n+1$. Let $\xi$ be a regular field on $E$ and $g$ a Riemannian metric on $E$ such that $\xi$ is a unit Killing vector field.

Since $\xi$ is regular and the manifold $E$ is compact the integral curves of $\xi$ are homeomorphic to $S^{1}$. 
This allows us to define an effective action without fixed points of $S^{1}$ on $E$ (Boothby and Wang [1]) and to construct in this way the principal circle bundle $E \rightarrow E / \xi=B$, where $B$ denotes the set of orbits, which is a differentiable manifold (Palais [5]).

Let $\omega$ be the dual form of $\xi$ by $g$, that is:

$$
\omega(X)=g(\xi, X) .
$$

Let us now introduce a Riemannian metric on $B$. For this we define

$$
h(X, Y)(p)=g\left(X^{\prime}, Y^{\prime}\right)(q)-\omega \otimes \omega\left(X^{\prime}, Y^{\prime}\right)(q)
$$

where $X^{\prime}, Y^{\prime}$ are fields on $E$, which are projected on $X, Y$ respectively, and $q$ is a point on the fibre of $p$.

It is easy to see that this definition is independent of the point $q$ and of the fields $X^{\prime}, Y^{\prime}$ chosen. We also see that it is a Riemannian metric. $\omega \otimes \omega$.

So, we have that $(B, h)$ is a Riemannian manifold with $g=\pi^{*} h+$

Moreover, since $E$ is orientable, there is a non-vanishing $2 n+1$ form on $E$. This form, integrated over the fibres (the bundle is orientable), is a non-vanishing $2 n$-form on $B$. So $B$ is orientable.

Therefore on $B$ we have:

$$
\int_{B} Q=2^{2 n} \pi^{n} n ! \chi(B)
$$

where $Q=(-1)^{n} \sum \varepsilon\left(i_{1} \cdots i_{2 n}\right) \Omega_{i_{1}}^{i_{2}} \wedge \cdots \wedge \Omega_{i_{2 n-1}}^{i_{2}}, \Omega_{j}^{i}$ denote the curvature forms of the metric $h$ and $\chi(B)$ is the Euler-Poincare characteristic of $B$.

Since $\omega(X)=g(\xi, X)$ and $\xi$ is a Killing vector field we have that $\omega$ is a connection form on the fibre bundle and so we can write $\Omega_{j}^{i}$ as a function of the curvature on $E$ with respect to the metric $g$ (Kobayashi).

Let $\psi_{\beta}^{\alpha}, \omega_{j}^{i}$ be the connection forms on $E$ and $B$, with respect to $g$ and $h$ respectively. Let $\Psi_{\beta}^{\alpha}, \Omega_{j}^{i}$ be the curvature forms of the connections defined by $\psi_{\beta}^{\alpha}$ and $\omega_{j}^{i}$. Set $\Gamma=d \omega$.

Let $U$ be a small open set in which $h=\sum_{j} \theta^{j} \otimes \theta^{j}$ where $\theta^{1}, \cdots, \theta^{2 n}$ are 1-forms defined on $U$. $\quad \Gamma=\pi^{*}\left(\sum_{\imath j} A_{i j} \theta^{i} \wedge \theta^{j}\right)$ with $A_{i j}+A_{j_{\imath}}=0$. Set $\varphi^{0}=\omega, \varphi^{i}=\pi^{*}\left(\theta^{i}\right)$ so that $g=\sum_{\alpha} \varphi^{\alpha} \otimes \varphi^{\alpha}$.

Connections and curvatures are related as follows:

$$
\begin{aligned}
& \psi_{0}^{0}=0 \\
& \psi_{0}^{i}=-\psi_{i}^{0}=-\sum_{j} A_{2 j} \varphi^{j} \\
& \psi_{j}^{i}=\pi^{*}\left(\omega_{j}^{i}\right)-A_{\imath j} \varphi^{0}
\end{aligned}
$$




$$
\begin{aligned}
& \Psi_{0}^{0}=0 \\
& \Psi_{0}^{i}=-\Psi_{i}^{0}=-\sum_{k s} A_{i k} A_{k s} \Phi^{s} \wedge \varphi^{0}-\sum_{k s} A_{l k ; s} \varphi^{s} \wedge \varphi^{k} \\
& \Psi_{j}^{i}=\pi^{*}\left(\Omega_{j}^{i}\right)-\sum_{k s}\left(A_{i j} A_{k s}+A_{i k} A_{j s}\right) \varphi^{k} \wedge \varphi^{s}-\sum_{k} A_{i j ; k} \varphi^{k} \wedge \varphi^{0}
\end{aligned}
$$

where $\sum_{k} A_{i j ; k} \theta^{k}=d A_{i j}-\sum_{k} A_{i k} \omega_{j}^{k}+\sum_{k} A_{k j} \omega_{k}^{j}$. It is easy to see that $A_{i j}=-(1 / 2)\left(g\left(\xi,\left[e_{i}, e_{j}\right]\right)\right)=-g\left(e_{i}, \nabla_{e_{j}} \xi\right)$.

Proposition 1. In this hypothesis, all trajectories of $\xi$ have the common length $\iota(\xi)$.

Proof. Since $\xi$ is a unit vector field we have, by means of its 1parameter group, a parametrization by arc-length. We set $\lambda(p)=$ inf $\left\{t \in R ; t>0\right.$ and $\left.\varphi_{t}(p)=p\right\}$ the period of the orbit through $p$. It is constant on each orbit. Clearly $\lambda(p)$ coincides with the length of the orbit through $p$.

Let $U$ be an open connected set, and $p \in U$. Let $V=\varphi_{t}\left((-\varepsilon, \varepsilon) \times V_{0}\right)$ be a neighborhood of $p . \varphi_{0}(p)=p$ with $V_{0}$ transversal with the 1parameter group. For $x \in V$, by taking a smaller neighborhood if need be, we may suppose that there exists an orthogonal curve $\sigma:[0,1] \rightarrow E$ with $\sigma(0)=p$ and $\sigma(1)=x^{\prime}$ where $x$ and $x^{\prime}$ lie on the same orbit.

Then, the transformed $\sigma^{\prime}$ of $\sigma$ by $\varphi_{\lambda(p)}$ is another orthogonal curve (since $\xi$ is a Killing field) with $\sigma(0)=\sigma^{\prime}(0)=p$ and $\pi \circ \sigma^{\prime}=\pi \circ \sigma$. Then by a straightforward application of the uniqueness of solutions of ordinary differential equations we have $\sigma=\sigma^{\prime}$ and so the period of $p$ coincides with the period of $x^{\prime}$.

By (4) we have

$$
\begin{gathered}
\left((-1)^{n} / 2^{2 n} \pi^{n} n ! \iota(\xi)\right) \int_{E} \sum \varepsilon\left(i_{1} \cdots i_{2 n}\right) \pi^{*} \Omega_{\imath_{1}}^{i_{2}} \\
\wedge \cdots \wedge \pi^{*} \Omega_{i_{2 n-1}^{i_{2 n}}} \wedge \varphi^{0}=\chi(B) .
\end{gathered}
$$

If we put $A(i j)$ instead of $A_{i j}$ we have

$$
\begin{aligned}
& \left((-1)^{n} / 2^{2 n} \pi^{n} n ! \ell(\xi)\right) \int_{E} \sum \varepsilon\left(i_{1} \cdots i_{2 n}\right)\left(\Psi_{i_{1}}^{i_{2}}\right. \\
& \left.\quad+\sum_{k s}\left(A\left(i_{2} i_{1}\right) A(k s)+A\left(i_{2} k\right) A\left(i_{1} s\right)\right) \varphi^{k} \wedge \varphi^{s}\right) \wedge \cdots \wedge\left(\Psi_{i_{2 n-1}}^{i_{2 n}}\right. \\
& \left.\quad+\sum_{k s}\left(A\left(i_{2 n} i_{2 n-1}\right) A(k s)+A\left(i_{2 n} k\right) A\left(i_{2 n-1} s\right)\right) \varphi^{k} \wedge \varphi^{s}\right) \wedge \varphi^{0} \\
& =\chi(B) .
\end{aligned}
$$

Let us compute $\chi(B)$ in terms of the Betti numbers of $E$. The exact Gysin sequence of the bundle $\pi: E \rightarrow B$ is

$$
0 \longrightarrow H^{1}(B) \stackrel{\pi^{\sharp}}{\longrightarrow} H^{1}(E) \stackrel{S_{S^{1}}^{*}}{\longrightarrow} H^{0}(B) \stackrel{D_{0}}{\longrightarrow} \cdots \text {. }
$$


Splitting it

$$
0 \rightarrow H^{p+2}(B) / \operatorname{Im} D_{p} \rightarrow H^{p+2}(E) \rightarrow \operatorname{Ker} D_{p+1} \rightarrow 0
$$

and so, since they are vector spaces, we have:

$$
H^{p+2}(E)=\operatorname{Ker} D_{p+1} \oplus \operatorname{Coker} D_{p} .
$$

Then we get the following relations:

$$
\begin{aligned}
b_{0}(B) & =b_{0}(E) \\
b_{1}(B) & =b_{1}(E)-d_{0} \\
b_{2}(B) & =b_{2}(E)+b_{0}(E)-d_{0}-d_{1} \\
& \vdots \\
b_{n}(B) & =b_{n}(E)+b_{n-2}(E)+\cdots+b_{n-2 k}(E)-d_{0}-d_{1}-\cdots-d_{n-1},
\end{aligned}
$$

(with $n=2 k$ or $n=2 k+1, d_{\imath}=\operatorname{dim} \operatorname{Ker} D_{i}$ and $D_{\imath}: H^{i}(B ; \boldsymbol{R}) \rightarrow H^{i+2}(B ; \boldsymbol{R})$ the multiplication by the Euler class of the bundle).

Since

$$
\chi(B)=\sum_{r=0}^{n-1} 2 b_{r}(B)(-1)^{r}+b_{n}(B)(-1)^{n} \quad(\operatorname{dim} B=2 n),
$$

expressing each $b_{r}(B)$ as a function of $b_{r}(E)$ and $d_{r}$ we have

$$
\chi(B)=\sum_{r=0}^{n}(n+1-r)(-1)^{r} b_{r}(E)+\sum_{r=0}^{n-1}(-1)^{r} d_{r} .
$$

In this way we get formula (3), with $f(\Omega, \xi)$ given by (8). This $f(\Omega, \xi)$ is independent of the choice of $\xi$-frame fields.

\section{Dimension 3.}

THEOREM 2. Let $(E, g)$ be a compact connected orientable Riemannian manifold of dimension 3 . Let $\xi$ be a regular unit Killing vector field on $E$. Then

$$
(1 / 2 \pi \iota(\xi)) \int_{E}\left(K\left(\xi^{\perp}\right)+3 K(\xi)\right) \eta=2-b_{1}(E)
$$

if and only if $b_{1}(E)$ is even, and

$$
(1 / 2 \pi \iota(\xi)) \int_{E}\left(K\left(\xi^{\perp}\right)+3 K(\xi)\right) \eta=3-b_{1}(E)
$$

if and only if $b_{1}(E)$ is odd, where $K\left(\xi^{\perp}\right)$ means the sectional curvature of the plane orthogonal to $\xi$, and $K(\xi)$ means the sectional curvature of any plane containing $\xi$ (it is independent of the choice of the plane) and $\eta$ denotes the volume form.

Moreover in the last case the bundle is trivial. (Then it is clear 
that the right hand side is independent of $\xi$, in the sense that if $\xi^{\prime}$ is another regular unit Killing vector field on $E$, then $\chi(E / \xi)=\chi\left(E / \xi^{\prime}\right)$.

Proof. $\chi(E / \xi)=2-b_{1}(E)+d_{0}$. $E / \xi$ is a Riemann surface, so $b_{1}(E / \xi) \equiv \chi(E / \xi) \equiv 0(\bmod 2)$. If $b_{1}(E)$ is even we have $d_{0}$ even and since $d_{0} \leqq b_{0}(E / \xi)=1$ (by connectedness) we have $d_{0}=0$. If $b_{1}(E)$ is odd we have $d_{0}$ odd and so $d_{0}=1$. By (8) we have

$$
\begin{aligned}
& (-1 / 4 \pi \iota(\xi)) \int_{E} 2\left(\Psi_{1}^{2}-3 A(12)^{2} \varphi^{1} \wedge \varphi^{2}\right) \wedge \varphi^{0} \\
& =(1 / 2 \pi \ell(\xi)) \int_{E}\left(K\left(\xi^{\perp}\right)+3 K(\xi)\right) \eta=\chi(B) .
\end{aligned}
$$

Let us compute $A(12)^{2}$. Let $X_{1}, X_{2}, \xi$ be an orthonormal frame. $R\left(\xi, X_{1}, \xi, X_{1}\right)=g\left(R\left(\xi, X_{1}\right) X_{1} ; \xi\right)=\Psi_{1}^{0}\left(\xi, X_{1}\right)=A(12) A(21) \varphi^{1} \wedge \varphi^{0}\left(\xi, X_{1}\right)=$ $A(12)^{2}$.

So $A(12)^{2}$ is the sectional curvature of the plane determinated by $\xi$ and $X_{1}$. If $Z=\lambda X_{1}+\mu X_{2}$ with $\lambda^{2}+\mu^{2}=1$ it is easy to see that $R(\xi, Z, \xi, Z)=A(12)^{2}$. So we can say that $A(12)^{2}=K(\xi)=$ the sectional curvature of any plane containing $\xi$.

Analogously we have $\Psi_{1}^{2} \wedge \varphi^{0}=-K\left(\xi^{\perp}\right) \varphi^{0} \wedge \varphi^{1} \wedge \varphi^{2}$.

So (11) and (12) are proved.

Tanno's theorem in dimension 3 is contained in (11) since for Sasakian manifolds we have $b_{1}(E)$ even and $K(\xi)=1$.

5. Dimension 5. In the proof of Theorem 2 we use the fact that $E / \xi$ is a Riemann surface, that is, an algebraic curve. If we want to do an analogous study in dimension 5, we would have to impose at least the condition that $E / \xi$ is a complex manifold.

For this, it is sufficient to assume that $E$ admits an almost contact normal structure from Morimoto's theorem [4].

THEOREM 3. Let $(E, g)$ be a connected compact Riemannian manifold of dimension 5 with a normal almost contact regular structure $(\varphi, \xi, \eta)$, $\xi$ unit Killing vector field. Then

$$
\left(1 / \ell(\xi) 2^{4} \pi^{2} 2 !\right) \int_{E} F(\Omega, \xi)=3-2 b_{1}(E)+b_{2}(E)
$$

if the Betti numbers of $E$ satisfy the $R_{1}$-condition. And

$$
\left(1 / \measuredangle(\xi) 2^{4} \pi^{2} 2 !\right) \int_{E} F(\Omega, \xi)=5-3 b_{1}(E)+b_{2}(E)
$$

if the Betti numbers of $E$ satisfy the $R_{2}$-condition.

It is obvious that the right hand side does not depend on the chosen 
unit Killing vector field. In this formula $F(\Omega, \xi)$ is given by (8) where $A(i j)=g\left(e_{i}, \nabla_{e_{j}} \xi\right), \phi^{k}$ dual of $e_{k}$, and $\xi, e_{1}, \cdots, e_{2 n}$ an orthonormal frame.

We prove Theorem 3 studying separately the different cases of conditions $R_{1}$ and $R_{2}$.

THEOREM 3a. Let $E$ be a compact connected orientable Riemannian manifold of dimension 5 , and $\xi$ a regular unit Killing vector field on $E$ such that $E / \xi$ is a complex manifold. Then, if $b_{1}(E)$ and $b_{2}(E)$ satisfy the $R_{2}$-condition, we have $E=E / \xi \times S^{1}$ and $\chi(E / \xi)=5-3 b_{1}(E)+b_{2}(E)$.

Proof. If the fibration is not trivial we have $d_{0}=0$ and so $b_{1}(B)=$ $b_{1}(E)$ odd $\neq 1$. By Grauert's theorem we have $B=Q \cdots Q\left(B^{\prime}\right)$, with $B^{\prime}$ free from exceptional curves (of the first kind). The geometric genus $p_{g}$ and the irregularity $q$ of $B$ are invariant under quadratic transformations. Thus $b_{1}(B)=b_{1}\left(B^{\prime}\right) \neq 1$ and odd. So by Theorem $B$ we have $c_{1}^{2}\left(B^{\prime}\right)=0$. But $c_{1}^{2}(B)+n_{t}=c_{1}^{2}\left(B^{\prime}\right)=0$ implies $c_{1}^{2}(B) \leqq 0$. Moreover $c_{1}^{2}(B)+$ $b^{-}(B)=c_{1}^{2}\left(B^{\prime}\right)+b^{-}\left(B^{\prime}\right)=b^{-}\left(B^{\prime}\right)$ implies $c_{1}^{2}(B)+b^{-}(B) \geqq 0$. From $c_{1}^{2}(B)+$ $b^{-}(B)=10 p_{g}(B)+8-8 q(B) \geqq 0$ we have $p_{g}(B) \geqq[(8 q(B)-8) / 10]+\delta_{01}$.

Moreover, from the Gysin exact sequence, we have $b_{2}(E)=b_{2}(B)-$ $b_{0}(E)+d_{0}+d_{1}$. And from Theorem A, $b_{2}(E)=b^{+}(B)+b^{-}(B)-1+d_{0}+$ $d_{1}=2 p_{g}(B)+b^{-}(B)-1+d_{1} \geqq 2 p_{g}(B)+b^{-}(B)-1$. Also from Theorem A we have:

$$
\begin{aligned}
& b_{2}(E) \geqq 12 p_{g}(B)+3-4 b_{1}(E), \quad \text { that is }, \\
& b_{2}(E) \geqq 12\left[\left(4 b_{1}(E)-4\right) / 10\right]+12 \delta_{01}+3-4 b_{1}(E),
\end{aligned}
$$

but we have excluded this case.

Thus the fibration must be trivial and therefore $d_{0}=1$ and $d_{1}=$ $b_{1}(E)-1$. So we have $\chi(B)=5-3 b_{1}(E)+b_{2}(E)$ independently of the unit Killing regular vector field.

THEOREM 3b. Let $E$ be a compact connected orientable Riemannian manifold of dimension 5, and $\xi$ a regular unit Killing vector field on $E$ such that $E / \xi$ be a complex manifold. Then if $b_{1}(E)$ is even, $b_{1}(E) \neq 2$, and $b_{2}(E)=0$ or $b_{2}(E)=1$, we have $E \neq E / \xi \times S^{1}$ and $\chi(E / \xi)=3$ $2 b_{1}(E)+b_{2}(E)$.

Proof. If the fibration is trivial, we have $d_{0}=1$ and $b_{1}(B)=$ $b_{1}(E)-1$. Therefore, $b_{1}(B)$ is odd and $b_{1}(B) \neq 1$. As in the above case, we have $B=Q \cdots Q\left(B^{\prime}\right)$ with $B^{\prime}$ free from exceptional curves. Similarly we have $c_{1}^{2}\left(B^{\prime}\right)=0$ and so $c_{1}^{2}(B)+b^{-}(B)=c_{1}^{2}\left(B^{\prime}\right)+b^{-}\left(B^{\prime}\right) \geqq 0$. This implies $p_{g}(B) \geqq\left[\left(4 b_{1}(E)-8\right) / 10\right]+\delta_{01}$ and $b_{2}(E) \geqq 2\left[\left(4 b_{1}(E)-8\right) / 10\right]+2 \delta_{01}$ and this inequality is not true for $b_{2}(E)=0$ or $b_{2}(E)=1$.

Thus the fibration must be non-trivial. In this case, $d_{0}=0$ and 
$b_{1}(B)=b_{1}(E)$ even different from 2. Since $b_{2}(B)=b_{2}(E)+1-d_{1}=$ $2 p_{g}(B)+1+b^{-}(B)$, if $b_{2}(E)=0$ we have $1-d_{1}=2 p_{g}(B)+1+b^{-}(B)$ and this implies $\chi(E / \xi)=3-2 b_{1}(E)$. This proves the theorem for $b_{2}(E)=0$.

If $b_{2}(E)=1$, let us see that $c_{1}^{2}\left(B^{\prime}\right)<0$ : Otherwise we have $c_{1}^{2}(B)+$ $b^{-}(B) \geqq 0$, and then $p_{g}(B) \geqq\left[\left(4 b_{1}(E)-9\right) / 10\right]+\delta_{01}$ by Theorem A. Also $b_{2}(E)=b_{2}(B)-1+d_{1} \geqq 2 p_{g}(B)$, and so $b_{2}(E) \geqq 2\left[\left(4 b_{1}(E)-9\right) / 10\right]+2 \delta_{01}$, and this inequality is not true for $b_{2}(E)=1$. Thus $c_{1}^{2}\left(B^{\prime}\right)<0$. Then, since $b_{1}\left(B^{\prime}\right)$ is even we are in the first five cases in the table of Theorem $\mathrm{B}$, and since $c_{1}^{2}\left(B^{\prime}\right)<0$, we are in the first case.

Since $c_{1}^{2}\left(P_{2}(C)\right)=9, B^{\prime}$ is a ruled surface, that is, $B^{\prime}=P_{1}(C) \times S$, where $S$ is a curve of genus $p\left(\operatorname{dim}_{c} S=1\right)$. Therefore $b_{2}\left(B^{\prime}\right)=b_{2}\left(P_{1}(C)\right)+$ $b_{1}\left(P_{1}(C)\right) \cdot b_{1}(S)+b_{2}(S)=1+1=2$, since $b_{1}(S)=2 p, \quad b_{0}(S)=1, \quad b_{2}(S)=1$ and $b_{1}\left(P_{1}(C)\right)=0, b_{0}\left(P_{1}(C)\right)=1, b_{2}\left(P_{1}(C)\right)=1$.

Moreover $b_{2}\left(B^{\prime}\right)=2$ implies $b^{-}(B)=2-b^{+}\left(B^{\prime}\right)=2-2 p_{g}\left(B^{\prime}\right)-1$ and this implies $p_{g}\left(B^{\prime}\right)=p_{g}(B)=0$ and $b^{-}\left(B^{\prime}\right)=1$. Therefore $b^{-}(B)=b^{-}\left(B^{\prime}\right)+$ $n_{t} \geqq 1$. From $b_{2}(E)+1-d_{1}=2 p_{g}(B)+1+b^{-}(B)$ we have $d_{1}=0$ and $\chi(E / \xi)=3-2 b_{1}(E)+1$. This proves the theorem.

In both cases we have proved that the surface $E / \xi$ is free from exceptional curves.

Let us study separately the cases 1,2 , and 3 of the condition $R_{1}$.

Proposition 2. Let $(E, \xi)$ be as in the above theorem. Then if $b_{1}(E)=2$ and $b_{2}(E)=0$ we have $\chi(E / \xi)=-1$ and the fibration is not trivial.

Proof. In this case $b_{2}(B)=1-\left(d_{0}+d_{1}\right) . \quad d_{0}=0$ implies $b_{1}(B)=$ $b_{1}(E)=2$ and so $b_{2}(B)=2 p_{g}(B)+1+b^{-}(B)$. This implies $d_{1}=0$ and $\chi(E / \xi)=-1 . \quad d_{0}=1$ implies $b_{1}(B)=d_{1}=b_{1}(E)-d_{0}=1$ and this implies $b_{2}(B)=1-\left(d_{0}+d_{1}\right)=1-2$ and this is impossible.

Proposition 3. Let $(E, \xi)$ be as in the above proposition. Then $b_{1}(E)=0$ implies $\chi(E / \xi)=3+b_{2}(E)$ and the fibration is not trivial.

Proof. $\quad b_{1}(B)=b_{1}(E)-d_{0}=-d_{0}$ implies $d_{0}=b_{1}(B)=0$. Since $d_{1} \leqq$ $b_{1}(B)$ we have $d_{1}=0$.

Proposition 4. Let $(E, \xi)$ be as in the above proposition. If $b_{1}(E)=2$ and $b_{2}(E)=1$, we have $\chi(E / \xi)=0$.

Proof. $\quad b_{2}(B)=1+1-\left(d_{0}+d_{1}\right) . \quad$ (i) $d_{0}=0$ implies $b_{1}(B)=2$ and $b_{2}(B)=2-d_{1}=2 p_{g}(B)+1+b^{-}(B)$ and this implies $d_{1}=0$ or 1 .

Let us see that $d_{1} \neq 1$. Assume $b_{2}(B)=1$, then $p_{g}(B)=b^{-}(B)=0$. But $b^{-}(B)=0$ implies that $B$ is free from exceptional curves (cf. proof 
of Theorem 3a). By Theorem $\mathrm{A}$, we have $c_{1}^{2}(B)=10 p_{g}+9-b^{-}-8 q=$ $1>0$ and $q=1$. But by Theorem $\mathrm{C}$, we have $q=0$. This is a contradiction. Thus $d_{1}=0$. (ii) If $d_{0}=1$, then $d_{1}=b_{1}(B)=b_{1}(E)-d_{0}=1$. In both cases $\chi(E / \xi)=3-2 b_{1}(E)+b_{2}(E)+d_{0}-d_{1}=3-2 b_{1}(E)+b_{2}(E)=0$.

Proposition 5. Let $(E, \xi)$ be as in the above proposition. If $b_{1}(E)=1$ and $b_{2}(E)=0$, we have $\chi(E / \xi)=1$ and the fibration is not trivial.

Proof. $\quad b_{2}(B)=1-\left(d_{0}+d_{1}\right)$. If $d_{0}=0, b_{1}(B)=b_{1}(E)=1$. Therefore $b_{2}(B)=2 p_{g}(B)+b^{-}(B)=b_{2}(E)+b_{0}(E)-d_{0}-d_{1}=1-d_{1}$. This implies $p_{g}(B)=0$ and $b^{-}(B)+d_{1}=1$. If $d_{1}=1, b^{-}(B)=0$ implies $b_{2}(B)=0$, but this is impossible, since $\chi_{E} \in H^{2}(B ; R)$ and $\chi_{E}$ is not zero because $d_{0}=0$. If $d_{0}=1, d_{1}=b_{1}(B)=b_{1}(E)-1=0$, but then $b_{2}(B)=0=2 p_{g}(B)+1+$ $b^{-}(B)$ and this is impossible. The last equality follows from Theorem A. Therefore, we have $d_{0}=0$ and $d_{1}=0$. So $\chi(E / \xi)=1$ and the fibration is not trivial.

Obviously, Theorem 3 follows from Theorems $3 a$ and $3 b$, and Propositions 2, 3, 4 and 5. The integrand is obtained from (8) for $n=2$.

We have obtained, too, the following theorem:

THEOREM 4. Let $E$ be a compact connected manifold of dimension 5 , with $b_{1}(E)$ even, different from zero and two, and $b_{2}(E)=0$, then $E$ cannot admit any almost contact normal regular structure.

Proof. In the proof of Theorem $3 \mathrm{~b}$, the existence of a field $\xi$ with $E / \xi$ being a complex manifold, implies the fibration being non-trivial and $p_{g}(B)=b^{-}(B)=0$. From this last equality we see that $B$ is free from exceptional curves.

Then, from Theorem A we would have $c_{1}^{2}(B)=9-8 q<0$, and using Theorem B, $B$ would be a ruled surface and so $b_{2}(B)=2=b^{+}(B)+b^{-}(B)=$ $2 p_{g}(B)+1+b^{-}(B)$, and this is impossible.

6. Higher dimensions. In higher dimensions we have obtained the following result:

THEOREM 5. Let $E$ be a compact connected Riemannian manifold of dimension $n=3+4 m, m>0$, which admits a regular unit Killing vector field $\xi$. If $b_{2}(E)=b_{4}(E)=\cdots=b_{2 m}(E)=0$, then

$$
\chi(E / \xi)=\sum_{r=0}^{2 m+1}(n+1-r)(-1)^{r} b_{r}(E)+1
$$

if and only if $b_{1}(E)+b_{3}(E)+\cdots+b_{2 m+1}(E)$ is odd. And 


$$
\chi(E / \xi)=\sum_{r=0}^{2 m+1}(n+1-r)(-1)^{r} b_{r}(E)
$$

if and only if $b_{1}(E)+b_{3}(E)+\cdots+b_{2 m+1}(E)$ is even.

$$
\text { If } b_{1}(E)=\cdots=b_{2 m+1}(E)=0 \text {. Then }
$$

$$
\chi(E / \xi)=\sum_{r=0}^{2 m+1}(n+1-r)(-1)^{r} b_{r}(E) .
$$

(Therefore, $\chi(E / \xi)$ is independent of the unit regular Killing vector field $\xi$.)

Proof. By Gysin exact sequence and the Poincaré duality we have the following relations:

$$
\begin{aligned}
& b_{1}(B)=b_{1}(E)-d_{0}=b_{4 m+1}(B)=b_{4 m+1}(E)+\cdots+b_{1}(E)-d_{0}-\cdots-d_{4 m} . \\
& b_{2}(B)=b_{2}(E)+1-d_{0}-d_{1}=b_{4 m}(E)+\cdots+b_{0}(E)-d_{0}-\cdots-d_{4 m-1} .
\end{aligned}
$$

Thus

$$
b_{4 m+1}(E)+\cdots+b_{3}(E)=d_{1}+\cdots+d_{4 m}
$$

and

$$
b_{4 m}(E)+\cdots+b_{4}(E)=d_{2}+\cdots+d_{4 m-1} .
$$

But $b_{4 m}(E)=b_{3}(E), \cdots$, and substracting (20) from (19) we have $b_{4 m+1}(E)=b_{2}(E)=d_{1}+d_{4 m}$. Analogously $b_{4}(E)=d_{3}+d_{4 m-2}$.

Therefore, it follows from hypothesis that $d_{1}=d_{3}=\cdots=d_{2 m-1}=0$. Moreover, if $\xi^{\prime}$ is another regular unit Killing vector field with $B^{\prime}=$ $E / \xi^{\prime}$, we have

$$
\begin{aligned}
& b_{2 m+1}(B)-b_{2 m+1}\left(B^{\prime}\right)=d_{0}^{\prime}-d_{0}+d_{2}^{\prime}-d_{2}+\cdots+d_{2 m}^{\prime}-d_{2 m} \\
& b_{2 m+1}(B)-b_{2 m+1}\left(B^{\prime}\right) \equiv 0(\bmod 2) .
\end{aligned}
$$

We also have

$$
b_{2 m}(B)=1-\left(d_{0}+\cdots+d_{2 m-1}\right) .
$$

By (21) and (22) we have

$$
d_{0}^{\prime}+d_{2}^{\prime}+\cdots+d_{2 m}^{\prime}=2 k+d_{0}+d_{2} \cdots+d_{2 m} \text {. }
$$

Then if $d_{0}=1$, by (23) we have $d_{2}=\cdots=d_{2 m}=0$ and so $d_{0}+\cdots+$ $d_{2 m}=1$. If $d_{0}=0$, by (23) we have $b_{2 m}(B)=0$ or $1, d_{2 m}=0$ or 1 and $d_{2}+\cdots+d_{2 m-2}=0$ or 1 . If $d_{2}+\cdots+d_{2 m-2}=0$, we have $d_{0}+\cdots+$ $d_{2 m}=0$ or 1 . If $d_{2}+\cdots+d_{2 m-2}=1$, we have $b_{2 m}(B)=1-1=0$ and so $d_{2 m}=0$, thus $d_{0}+\cdots+d_{2 m}=0$ or 1 .

Analogously, $d_{0}^{\prime}+\cdots+d_{2 m}^{\prime}=0$ or 1 . By (24) we have $d_{0}^{\prime}+\cdots+d_{2 m}^{\prime}=$ $d_{0}+\cdots+d_{2 m}$. Therefore, $\chi(E / \xi)=\chi\left(E / \xi^{\prime}\right)=\sum_{r=0}^{n}(n+1-r)(-1)^{r} b_{r}(E)+$ 
$d_{0}+\cdots+d_{2 m}$. Since $b_{2 m+1}(B)=b_{2 m+1}(E)+\cdots+b_{1}(E)-\left(d_{0}+\cdots+d_{2 m}\right)$ is even, we have proved (17) and (18). and so:

If $b_{1}(E)=\cdots=b_{2 m+1}(E)=0$, it follows that $b_{2 m+1}(B)=-\left(d_{0}+\cdots+d_{2 m}\right)$

$$
\chi(E / \xi)=\sum_{r=0}^{n}(n+1-r)(-1)^{r} b_{r}(E) .
$$

This proves Theorem 5 .

CoRollary 1. Let $(E, \xi)$ be a compact connected Sasakian manifold of dimension 7 , with $\xi$ regular, whose curvature is strictly positive and $\xi^{\prime}$ another unit Killing regular vector field on $E$. Then $\chi(E / \xi)=\chi\left(E / \xi^{\prime}\right)$.

Proof. For this manifold $b_{2}(E)=0$. (Tanno [6]).

CoROLlaRY 2. $S^{2 p+1} \times S^{2 q}(p+q=2 m+1, p \leqq m<q)$ does not admit a regular Sasakian structure.

Proof. The Betti numbers of $S^{2 p+1} \times S^{2 q}(p+q=2 m+1, p \leqq m<q)$ satisfy $b_{2}=\cdots=b_{2 m}=0$ and $b_{1}+\cdots+b_{2 m+1}$ odd. So if $\xi$ is a regular unit Killing vector field on $S^{2 p+1} \times S^{2 q}$ we have

$$
\chi(E / \xi)=\sum_{r=0}^{2 m+1}(n+1-r)(-1)^{r} b_{r}(E)+1
$$

and therefore $\xi$ is not Sasakian.

Nevertheless $S^{2} \times S^{3}\left(S^{6} \times S^{7}\right)$ admits a Sasakian regular structure.

7. Remarks. (i) Let $(E, g)$ be as in Theorem 2. If $(E, g)$ is of constant curvature $k$, we have $k \geqq 0$ and:

If $k=0$, then $E$ is homeomorphic to $S^{1} \times S^{1} \times S^{1}$.

If $k>0$, then $E$ is a fibre bundle on $S^{2}$.

If $(E, g)$ is not of constant curvature but $K(\xi)=k \neq 0$, then $\left(E, g^{\prime}, \xi^{\prime}\right)$ with $g^{\prime}=k g$ and $\xi^{\prime}=\xi / \sqrt{k}$ is a Sasakian manifold.

(ii) Let $(E, g)$ be as in Theorem 3. We want to know in which cases the integrand of (14) (or (15)) is positive. We know that the integrand of the Gauss-Bonnet formula in dimension 4 can be written as follows (Chern, Abh. Math. Sem. Univ. Hamburg, 20 (1955), p. 124):

$$
\begin{aligned}
& \left(K_{1212} K_{3434}+K_{1234}^{2}+K_{1313} K_{4242}\right. \\
& \left.\quad+K_{1342}^{2}+K_{1414} K_{2323}+K_{1423}^{2}\right) \theta^{1} \wedge \cdots \wedge \theta^{4},
\end{aligned}
$$

where

$$
\Omega_{j}^{i}=(1 / 2) \sum_{k, r} K_{i j k r} \theta^{k} \wedge \theta^{r}
$$




$$
\Psi_{\beta}^{\alpha}=(1 / 2) \sum_{\lambda, \mu} R_{\alpha \beta \lambda \mu} \varphi^{\lambda} \wedge \Phi^{\mu}, \text { by }(6)
$$

we have

$$
R_{i j i j}=K_{i j i j}-3 A(i j)^{2} .
$$

Then, by (25) and (26), we have the following result: "If the sectional curvature is always positive, the integrand is positive. If the sectional curvature along the planes orthogonals to $\xi$ is always positive, the integrand is positive".

\section{REFERENCES}

[1] W. M. Boothby and H. C. Wang, On Contact manifolds, Annals of Math., 68 (1958), 721-734.

[2] S. KobayASHI, Topology of positively pinched Kaehler manifolds, Tôhoku Math. J., 15 (1963), 121-139.

[3] K. KodaIra, On the structure of compact complex analytic surfaces, I, II, III, IV, Amer. J. Math., 86 (1964), 751-798; 88 (1966), 682-721; 90 (1968), 55-83; 90 (1968), 1048-1066.

[4] A. MоRiмото, On normal almost contact structure with a regularity, Tôhoku Math. J., 16 (1964), 90-104.

[5] R. Palais, A global formulation of the Lie theory of transformation groups, Memoirs of the Amer. Math. Soc., 22 (1957).

[6] S. Tanno, The topology of contact Riemannian manifolds, Illinois J. Math., 12 (1968), 700-717.

[7] S. TANNo, A formula on some odd-dimensional Riemannian manifolds related to the Gauss-Bonnet formula, J. Math. Soc. Japan, 24 (1972), 204-212.

Departament De Geometria i Topologia

Universitat Autonoma de Barcelona

Bellaterra, Barcelona, Espanya 